\title{
Plasma heating in the initial phase of solar flares
}

\author{
P. Rudawy ${ }^{1}$, M. Siarkowski ${ }^{2}$ and R. Falewicz ${ }^{1}$ \\ ${ }^{1}$ Astronomical Institute, University of Wrocław, 51-622 Wrocław, ul. Kopernika 11, Poland \\ email: rudawy; falewicz@astro.uni.wroc.pl \\ ${ }^{2}$ Space Research Centre, Polish Academy of Sciences, 51-622 Wrocław, ul. Kopernika 11, \\ Poland \\ email: ms@cbk.pan.wroc.pl
}

\begin{abstract}
In this paper we analyze soft and hard X-ray emission of the 2002 September 20 M1.8 GOES class solar flare observed by RHESSI and GOES satellites, where soft X-ray emission precedes the onset of the main bulk hard X-ray emission by $\sim 5 \mathrm{~min}$. This suggests that an additional heating mechanism may be at work at the early beginning of the flare. However RHESSI spectra indicate presence of the non-thermal electrons also before impulsive phase. So, we assumed that a dominant energy transport mechanism during rise phase of solar flares is electron beam-driven evaporation. We used non-thermal electron beams derived from RHESSI spectra as the heating source in a hydrodynamic model of the analyzed flare. We showed that energy delivered by non-thermal electron beams is sufficient to heat the flare loop to temperatures in which it emits soft X-ray closely following the GOES 1-8 $\AA$ light-curve.
\end{abstract}

Keywords. Sun: flares, Sun: X-rays, gamma rays

\section{Introduction}

There are several papers that investigate temporal dependencies between the beginnings of SXR and HXR emissions. On average the SXR emission starts 3 min before the hard X-ray emission (see Machado et al. 1986, and Veronig et al. 2002).

An enhanced thermal emission preceding the onset of the hard X-rays may be indicative of a thermal preheating phase prior to the impulsive electron acceleration. Current-sheet models (e.g., Heyvaerts et al. 1977) of solar flares predict a preheating phase. Li et al. (1987) discussed the preheating phase of solar flares triggered by new emerging magnetic flux.An alternative mechanism to electron beam-driven evaporation, namely conducted driven evaporation, was developed lately by Battaglia et al. (2009). They explain the time evolution of the observed emission for all analyzed events as an effect of saturated heat flux. The tendency of the soft X-ray flux to appear before hard X-rays emission can be attributed also to the sensitivity threshold of the hard X-ray detectors Dennis (1988).

In this paper we show, using unprecedented high sensitivity of the RHESSI detectors Lin et al. (2002) and a numerical model of flare, that early SXR emission observed prior to impulsive phase could be fully explained without any ad-hoc assumptions (at least for analyzed event). All necessary energy to explain the soft emission could be derived from observed HXR spectra.

\section{Observations and modeling of the heating of the loop}

For our work we selected RHESSI observations obtained without the activation of the attenuators in order to prevent discontinuities in fitting parameters. A solar flare with a simple single-loop structure was then chosen for convenience in numerical modeling. The 
investigated flare occurred in active region NOAA 10126 (S23E69) on 2002 September 20. It was classified as an M1.8 GOES class flare. The SXR emission started to increase slowly at 09:18:15 UT (see Fig. 1). The RHESSI data were analyzed using RHESSI OSPEX package of the SolarSoftWare system. Data were summed over the front segments of the seven detectors with detectors number 2 and 7 excluded. The spectra were measured with 4 sec temporal resolution in 158 energy bands from 4 to $300 \mathrm{keV}$. We applied the energy widths $d E=0.3 \mathrm{keV}$ within the range $4-15 \mathrm{keV}, d E=1.0 \mathrm{keV}$ in the range $15-100 \mathrm{keV}$, and $d E=5.0 \mathrm{keV}$ above $100 \mathrm{keV}$. The analyzed spectra were corrected for pulse pile-up, decimation and albedo effects.
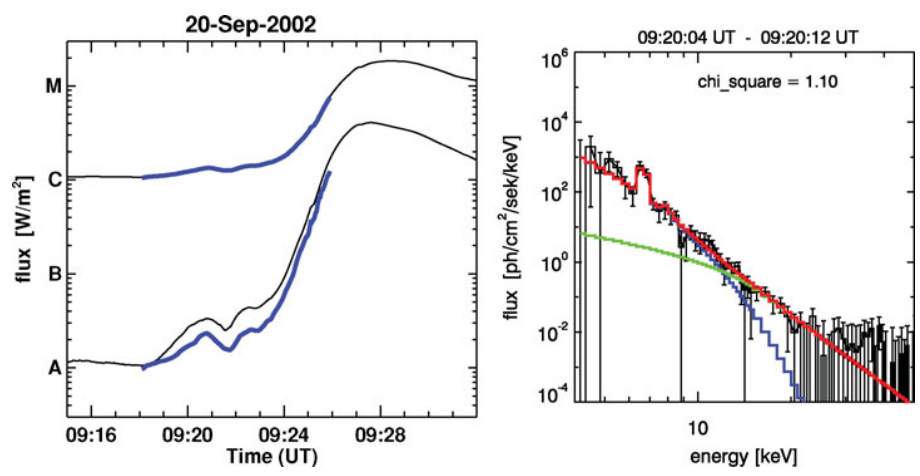

Figure 1. Left panel: RHESSI spectral fit results for data accumulated with a 8 sec time interval between 09:20:04-09:20:12 UT. The spectrum was fitted with one temperature thermal model (blue color) and thick target model (green color) with energy cut-off $E_{c}=15.8 \mathrm{keV}$. Total fitted spectrum is shown in red color. Right panel: Comparison of the observed (black thin lines) and calculated (thick blue line) GOES fluxes in 1-8 $\AA$ (upper curves) and $0.5-4$. $\AA$ (lower curves) energy bands during initial phase of the analyzed flare.

The spectra were fitted with single temperature thermal plus thick-target models. The thermal model was based on the X-ray continuum and line emission calculated by the CHIANTI atomic code. Figure 1 shows the fit of the spectrum, accumulated between 09:20:04 UT and 09:20:12 UT. In addition to the thermal component, there is an important power law shape for emission between $15 \mathrm{keV}$ and $20 \mathrm{keV}$ which can be recognized and fitted as thick-target emission of the non-thermal electrons.

In this work we used 1D Naval Research Laboratory Solar Flux Tube Model code by Mariska and his co-workers (Mariska et al. 1982, Mariska et al. 1989), slightly modified by us. We included: new radiative loss and heating functions, the VAL-C model of the initial structure of the lower part of the loop, and double precision calculations. For details see the paper by Falewicz et al. (2009).

We modeled the evolution of the analyzed flare as follows: an initial, quasi-stationary pre-flare models of the flaring loop was built using geometrical and thermodynamic parameters estimated from RHESSI and GOES data. These initial parameters of the flaring loop were as follows: semi-length $9500 \mathrm{~km}$, radius $1900 \mathrm{~km}$, pressure in feet $22 \mathrm{dyn} / \mathrm{cm}^{2}$. Small volumetric heating was used to keep this model in the quasi stationary state on the pre-flare level of activity before the start of the non-thermal heating. Then we started to model the heating of the loop by non-thermal electrons adding a dose of energy and calculating the resulting GOES flux. We used thick target parameters $N_{n t h}, \delta$ and $E_{c}$ obtained from fits of consecutive RHESSI spectra for each time-step as input into the Fisher's heating function. However, an acceptable fit can be obtained for all $E_{c}$ values in the range 5-30 keV for all following spectra. Because the low energy cut-off determines 
an amount of energy delivered to the loop, this non-uniqueness could be limited using an independent energetic condition, like observed 1-8 A GOES flux. Indeed, for each time step we adjusted $E_{c}$ values in order to achieve conformity of the observed and modeled fluxes in GOES 1-8 $\AA$ band. Such use of GOES 1-8 A flux put important limitation on the allowed low energy cut-off values and reduces importantly the non-uniqueness problem.

The final result of our modeling is presented in Figure 1 - right panel. The synthesized GOES 1-8 $\AA$ light-curve closely follows the observed one. The correspondence between observed and calculated fluxes in the case of the $0.5-4 \AA$ band is not so ideal but as a result we fully restored the observed slow increase of the SXR flux recorded before impulsive heating using only non-thermal electrons characteristics derived from the observed HXR spectra. Thus we confirmed that in the analyzed flare variations of the SXR and HXR fluxes are consistent with the Neupert effect.

\section{Conclusions}

We showed that it is possible to fit soft (GOES) and hard (RHESSI) X-ray emissions of a solar flares well before beginning of the impulsive phase without any additional heating besides the heating by non-thermal electrons. This was made possible because of the unprecedented high sensitivity of the RHESSI detectors which are able to measure very low hard X-ray flux early in the flare. Part of the emission, mainly in the energy range $<25 \mathrm{keV}$, is non-thermal in nature and indicates the presence of non-thermal electrons. In this case of a M1.8 GOES class flare, the non-thermal electron energy fluxes of the order of $10^{26} \mathrm{ergs} / \mathrm{sec}$, derived under the thick target interpretation, fully explains the required heating of the plasma and resulting increase in SXR emission.

Our results extend the standard model of SXR and HXR relationship to the early phases of solar flares and thus expands the number of flares consistent with the Neupert effect. These results also indicates that the process of electrons acceleration appears during the early stage of the flare, well before the impulsive phase.

Our method of adjustment of the low energy cut-off $E_{c}$ in order to equalize synthesized and observed GOES fluxes in 1-8 $\AA$ channel can be considered as a new method of $E_{c}$ determination.

\section{Acknowledgments}

The authors acknowledge the RHESSI consortium. This work was supported by the Polish Ministry of Science and Higher Education, grant number N203 022 31/2991.

\section{References}

Battaglia, M., Fletcher, L., \& Benz, A. O. 2009, A\&AA, 498, 891

Dennis, B. R. 1988, Solar Phys., 118, 49

Falewicz, R., Rudawy, P., \& Siarkowski, M. 2009, A\&A, 500, 901

Heyvaerts, J., Priest, E. R., \& Rust, D. M. 1977, ApJ, 216, 123

Li, H.-W., Pallavicini, R., \& Cheng, C. 1987, Solar Phys., 107, 271

Lin, R. P., Dennis, B. R., Hurford, G. J., et al. 2002, Solar Phys., 210, 3

Machado, M. E., Orwig, L. E., \& Antonucci, E. 1986, Adv. Space Res., 6(6), 101

Mariska, J. T., Boris, J. P., Oran, E. S., Young, T. R. Jr., \& Doschek, G. A. 1982, ApJ, 255, 738

Mariska, J. T., Emslie, A. G., \& Li, P. 1989, ApJ, 341, 1067

Neupert, W. M. 1986, ApJ, 153, L59

Veronig, A., Vrsnak, B., Temmer, M., \& Hanslmeier, A. 2002, Solar Phys., 208, 297 